\title{
In Vivo Reconstruction of Lumbar Erector Spinae Architecture Using Diffusion Tensor MRI
}

Citation for published version (APA):

Sieben, J. M., van Otten, I., Lataster, A., Froeling, M., Nederveen, A. J., Strijkers, G. J., \& Drost, M. (2016). In Vivo Reconstruction of Lumbar Erector Spinae Architecture Using Diffusion Tensor MRI. CLINICAL SPINE SURGERY, 29(3), E139-E145. https://doi.org/10.1097/bsd.0000000000000036

Document status and date:

Published: 01/01/2016

DOI:

10.1097/bsd.0000000000000036

Document Version:

Publisher's PDF, also known as Version of record

Document license:

Taverne

Please check the document version of this publication:

- A submitted manuscript is the version of the article upon submission and before peer-review. There can be important differences between the submitted version and the official published version of record.

People interested in the research are advised to contact the author for the final version of the publication, or visit the DOI to the publisher's website.

- The final author version and the galley proof are versions of the publication after peer review.

- The final published version features the final layout of the paper including the volume, issue and page numbers.

Link to publication

\footnotetext{
General rights rights.

- You may freely distribute the URL identifying the publication in the public portal. please follow below link for the End User Agreement:

www.umlib.nl/taverne-license

Take down policy

If you believe that this document breaches copyright please contact us at:

repository@maastrichtuniversity.nl

providing details and we will investigate your claim.
}

Copyright and moral rights for the publications made accessible in the public portal are retained by the authors and/or other copyright owners and it is a condition of accessing publications that users recognise and abide by the legal requirements associated with these

- Users may download and print one copy of any publication from the public portal for the purpose of private study or research.

- You may not further distribute the material or use it for any profit-making activity or commercial gain

If the publication is distributed under the terms of Article $25 \mathrm{fa}$ of the Dutch Copyright Act, indicated by the "Taverne" license above, 


\title{
In Vivo Reconstruction of Lumbar Erector Spinae Architecture Using Diffusion Tensor MRI
}

\author{
Judith M. Sieben, PhD, ${ }^{*} \dagger$ Ilse van Otten, MSc, $\$$ S Arno Lataster, MSc, ${ }^{*} \dagger$ Martijn Froeling, PhD, $\|$ \\ Aart J. Nederveen, PhD, $\mid$ Gustav J. Strijkers, PhD, /| and Maarten R. Drost, PhD\$\#
}

\begin{abstract}
Study Design: Diffusion tensor magnetic resonance imaging (DTMRI) reconstruction of lumbar erector spinae (ES) compared with cadaver dissection.

Objective: The aim of this study was to reconstruct the human lumbar ES from in vivo DT-MRI measurements and to compare the results with literature and cadaver dissection.

Summary of Background Data: DT-MRI enables 3-dimensional in vivo reconstruction of muscle architecture. Insight in ES architecture may improve the understanding of low back function. Furthermore, DT-MRI reconstructions allow individualized biomechanical modeling, which may serve as a clinical tool in injury evaluation and in improvement of understanding of pathologies like scoliosis.
\end{abstract}

Materials and Methods: The lumbar spine of 1 healthy male volunteer was scanned using a 3.0 T clinical MRI scanner. MRI data acquisition consisted of 3 parts: (1) high-resolution T1weighted turbo spin echo for anatomical reference; (2) DT-MRI measurements for fiber tractography; (3) dual echo gradient echo sequence for signal correction purposes. After processing, DT-MRI data were exported to a custom-built software program for fiber tractography. The resulting reconstructions were anatomically validated by comparison with cadaver dissection and literature.

Results: DT-MRI reconstruction of 4 parts of the lumbar ES (thoracic part of iliocostalis lumborum, lumbar part of iliocostalis lumborum, thoracic part of longissimus thoracis, and lumbar part of longissimus thoracis) adequately reflected its complex geometry. Some inaccuracies were found in reconstruction details. DT-MRI reconstructions were generally in

Received for publication June 3, 2013; accepted October 3, 2013.

From the *Department of Anatomy \& Embryology, Maastricht University; †CAPHRI School for Public Health and Primary Care, Maastricht; $\$$ Department of Radiology, Tweestedenziekenhuis, Tilburg; §Department of Human Movement Science, Maastricht University, Maastricht; $\|$ Biomedical NMR, Department of Biomedical Engineering, Eindhoven University of Technology, Eindhoven; -Department of Radiology, Amsterdam Academic Medical Center, Amsterdam; and \#NUTRIM School for Nutrition, Toxicology and Metabolism, Maastricht, The Netherlands.

The authors declare no conflict of interest.

Reprints: Judith M. Sieben, PhD, Department of Anatomy \& Embryology, Maastricht University, P.O. Box 616, 6200 MD Maastricht, The Netherlands (e-mail: j.sieben@maastrichtuniversity.nl).

Copyright (C) 2013 Wolters Kluwer Health, Inc. All rights reserved. agreement with anatomical descriptions from literature and with findings in a dissected cadaver specimen.

Conclusions: DT-MRI enables anatomically valid reconstruction of ES architecture. However, for reliable reconstruction of the smallest fascicles and attachments a higher resolution or application of higher-order models is needed. Reconstructions can be used as input for estimation of muscle architecture parameters in individualized biomechanical modeling. Such models are promising as a tool in clinical evaluation and in research of low back pain mechanisms.

Key Words: diffusion tensor imaging, erector spinae anatomy, skeletal muscle architecture, fiber tractography

(Clin Spine Surg 2016;29:E139-E145)

$\mathrm{D}$ iffusion tensor magnetic resonance imaging (DTMRI) has developed as a valuable addition to the spectrum of imaging techniques suitable to examine muscle anatomy. DT-MRI is especially useful as a method to depict muscle architecture, ${ }^{1-4}$ which is the arrangement of muscle fibers and connective tissue relative to the muscle's line of action. Muscle architecture is described by the macroscopic geometric properties of skeletal muscle, including parameters like fiber length, pennation angle, and physiological cross-sectional area. ${ }^{5}$ These features define a muscle's biomechanical functioning (ability to produce force and generate movement). Insight in muscle architecture is crucial in understanding normal muscle function and is of clinical relevance in diagnosis and treatment of muscle injuries and (neuro-) muscular pathologies. ${ }^{6-11}$ DT-MRI is a noninvasive imaging method that enables 3-dimensional (3D) in vivo reconstruction of a subject's personal muscle architecture, which allows estimation of specific, individual parameters that can be used in biomechanical modeling. Such models are of interest to both clinicians (eg, orthopedists) and researchers in the field of biomechanics and movement sciences. $^{12}$

The technique of DT-MRI is based on in vivo water diffusion characteristics in fibrous tissues like brain white matter, heart muscle, or skeletal muscle. ${ }^{12,13}$ Skeletal muscle tissue consists of very long (up to $30 \mathrm{~cm}$ ), cylindrical multinucleated cells (fibers) with a diameter of $10-100 \mu \mathrm{m}$, arranged in a regular, parallel pattern. ${ }^{14}$ Water will diffuse more easily in the direction of the fibers 
than perpendicular to it. This diffusion anisotropy may be due to various factors, including the presence of physical barriers like cell membranes, contractile proteins, and organelles. ${ }^{15-17}$ By measuring diffusion rates in 6 or more noncollinear directions, a diffusion tensor can be calculated, which is a mathematical representation of the diffusion that provides information about the directions of diffusion and their magnitude. The direction of the largest diffusivity coincides with the longitudinal axis of the cell, and thus with the predominant fiber orientation. ${ }^{18}$ A vector representing this orientation is derived for each MRI voxel (volumetric pixel). From these data, 3D fiber tracts can be reconstructed using fiber tractography algorithms. ${ }^{13}$ From these fiber reconstructions in vivo muscle architecture parameters (pennation angle, fiber length, physiological cross-sectional area) can be estimated. 1,19

First studies on skeletal muscle reconstruction using DT-MRI focused on lower limb musculature. ${ }^{2,15,20}$ More recently, DT-MRI has been used to study the muscles of the human forearm $4,21,22$ and to reconstruct the muscles of the pelvic floor. ${ }^{23,24}$ The current study focuses on the lumbar erector spinae (ES), which has a complex architecture, with a multitude of origins and insertions and many fiber trajectories of variable length and volume. In literature various descriptions of ES anatomy can be found ${ }^{25-29}$; consensus on the exact architecture is still lacking.

The aim of the present study was to segment and reconstruct the geometrically complex human lumbar ES from in vivo measurements using DT-MRI. The resulting reconstructions are anatomically validated by comparison with dissection of a cadaver specimen.

\section{MATERIALS AND METHODS}

For this study previously developed data acquisition and preprocessing protocols were used ${ }^{4}$; only the major aspects are highlighted in this paragraph.

\section{DT-MRI Acquisition and Muscle Fiber Reconstruction}

\section{Subject}

The images presented in this paper are derived from a healthy 29 -year-old male volunteer (body mass $85 \mathrm{~kg}$ ).

\section{Ethical Approval}

The institutional medical ethics review committee has considered the protocol. They stated that, according to the medical research involving human subjects act, ethical approval is not needed for this study. The subject consented on the use of the anonymized data for this study.

\section{Subject Positioning in Scanner}

Measurements were performed using a 3.0 T Philips Intera clinical MRI scanner (Philips Healthcare, Best, The Netherlands). The subject was placed in the scanner in a supine position on a 6-channel spine coil. The scan range reached from the level of the second sacral vertebra/posterior superior iliac spine to the 12th thoracic vertebra.

\section{MRI Protocol}

The MRI data acquisition consisted of 3 parts: (1) high-resolution T1-weighted turbo spin echo imaging for anatomical reference; (2) DT-MRI measurements for fiber tractography; and (3) a dual echo gradient echo sequence to allow correction for field inhomogeneities. All 3 data sets were acquired as stacks of 60 slices, with a slice thickness of $5 \mathrm{~mm}$. Additional imaging parameters can be found in appendix.

\section{Postprocessing}

For further processing MRI data were imported in Wolfram Mathematica 7. ${ }^{30}$ Background noise was removed from the diffusion-weighted images and signals were corrected for deformations (field inhomogeneities). Next, diffusion tensor values were estimated. A detailed description of the postprocessing computations can be found in Froeling et al. ${ }^{4}$

\section{Muscle Fiber Reconstruction}

Subsequently, the tensor data were exported to the DTITool software program for fiber tractography. ${ }^{31,32}$ For each muscle of the ES region of interest contours (ROIs) were drawn along the belly of the muscle. The fiber tractography algorithm computed the fiber tracts starting at the ROI and continuing bidirectionally until the stopping criterion (angle change $>10$ degrees per integration step) was met. ROIs were adjusted or added and fiber tract computation was repeated until the reconstruction included only the trajectories of the muscle of interest. Five to 10 iterations were needed to derive a reconstruction resembling the global shape of the muscle as depicted in the T1-weighted images.

\section{Background}

To provide anatomical reference for the reconstructed trajectories, a 3D reconstruction of the bony structures in the lumbar spine region was created using the Volume Tool software. ${ }^{33,34}$ Volumes of interest corresponding with the contours of the vertebrae, ilium, sacrum, and ribs were outlined in all consecutive transversal T1-weighted MRI slices. These volumes of interest data were then imported in the DT-MRI tool software and were visualized using 3D isosurfaces. This background facilitated verification of the muscle fiber attachment sites.

\section{Anatomical Validation}

The generally accepted division of ES in 4 parts as proposed by Bogduk ${ }^{26}$ was used as a theoretical framework for the DT-MRI fiber tractography reconstructions. Figure 1 shows a transverse plane T1MRI image at the level of the second lumbar vertebra, showing the topographical location of each of these muscle parts. The lateral part of ES consists of the 


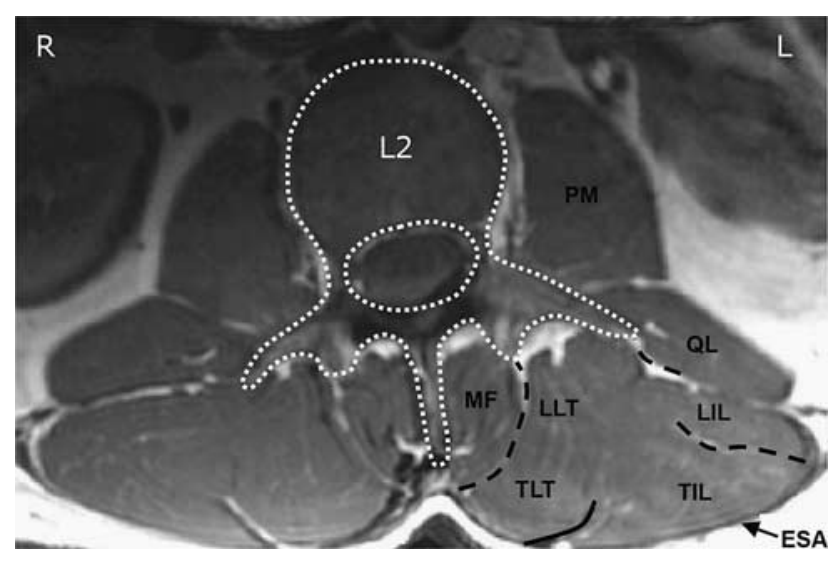

FIGURE 1. High-resolution T1-weighted transverse plane MRI at the level of the second lumbar vertebra. White dotted contour is that of L2 (second lumbar vertebra). Solid black lines indicate locations of aponeuroses (ESA, erector spinae aponeurosis); striped black lines indicate gliding planes (fat tissue) between muscles (PM, psoas major; $\mathrm{QL}$, quadratus lumborum). LIL indicates lumbar part of iliocostalis lumborum; LLT, lumbar part of longissimus thoracis; MF, multifidus; TIL, thoracic part of iliocostalis lumborum; TLT, thoracic part of longissimus thoracis.

superficially located thoracic part of iliocostalis lumborum (TIL) and the lumbar part of iliocostalis lumborum (LIL) underneath. The thoracic part of longissimus thoracis (TLT) overlying the deep lumbar part of longissimus thoracis (LLT) together make up the medial part (superficial and deep) of ES.

The resulting DT-MRI fiber tractography reconstructions were afterwards compared with several, in part contradictory, anatomical descriptions from literature. $^{25-29}$ In addition, dissection of a human adult cadaver was performed to visually validate the DT-MRI reconstructions.

The fiber trajectory reconstructions were performed by 1 researcher (I.v.O.), assisted by the study supervisor (M.R.D.). The anatomical dissection and comparison of the reconstructions with literature and dissection was performed by 2 clinical anatomists (J.M.S. and A.L.).

\section{Cadaver Dissection}

An embalmed (formalin fixed) adult human cadaver (female, age 77) was used for dissection. First, skin and subcutis were removed. Next, connective tissue, muscles and fat overlying the lumbar ES were carefully removed, leaving the ES themselves in place. In addition, the same bony structures as used for the background images were exposed. To facilitate comparison between fiber tractography reconstructions and dissection, paper markers were attached to the 11th and 12th ribs, spinous processes of the 12th thoracic (T12) and all lumbar vertebrae (L1-L5), median sacral crest at the level of the first sacral segment (S1), body of the second lumbar vertebra (V2), and posterior superior iliac spine $(\mathrm{P})$ in the dissected specimen.

\section{Photography}

Photographs were taken of the ES in situ from the same planes of view as used in the DT-MRI reconstructions. First, after removal of the overlying tissue, superficial parts of ES were photographed. Next, superficial parts were partly detached and folded out of view to visualize the deeper parts of ES.

\section{RESULTS}

DT-MRI trajectory reconstructions and dissections were performed for both sides of the body, which showed comparable results. The DT-MRI scan (positioning of the body inside the scanner) was optimized for the right side. Fiber tractography results for the right side of the body are presented in this section.

Trajectories are color coded for mean diffusivity (MD); high MD is shown in red (found in the muscular tissue that muscle bellies consist of) and low MD in pink or blue (found in connective tissue in the tendons and aponeuroses).

\section{TIL Reconstruction}

Fiber tractography results of TIL are shown in Figures 2B and C. Diffusivity was higher in the muscle bellies and lower in the tendinous parts that contribute to the lateral part of the erector spinae aponeurosis (ESA). Figures 2A and $\mathrm{D}$ show a comparable anatomical topography. With cranial attachments to the ribs and a caudal attachment to the iliac crest, TIL as a whole spans the lumbar region. However, the reconstruction shows a cranial attachment to the 11th rib only, because the more cranial attachments of TIL were outside the MRI-scan range.

\section{LIL Reconstruction}

LIL comprises of overlapping fascicles with cranial attachments to the L1-L4 transverse processes and the middle layer of the thoracolumbar fascia. Diffusivity coloring in LIL ventral view indicates large muscle bellies and short caudal tendons. (Images including LIL fiber tractography results and anatomical photographs are available from the corresponding author upon request.)

In Figure 3 close-ups of the trajectories of LIL L3 and L4 fascicles are presented to illustrate the ability to segment smaller details. The attachment of the fascicles to the tips of the transverse process of L3 and L4 can be distinguished. However, several trajectories of LIL do not connect entirely to the 3D-visualization of the vertebrae.

\section{TLT Reconstruction}

Reconstruction of cranial parts of TLT, like in TIL, was limited by MRI-scan range. Fiber tractography results for TLT showed several muscle bellies, arising from the consecutive vertebral levels, passing into long caudal 

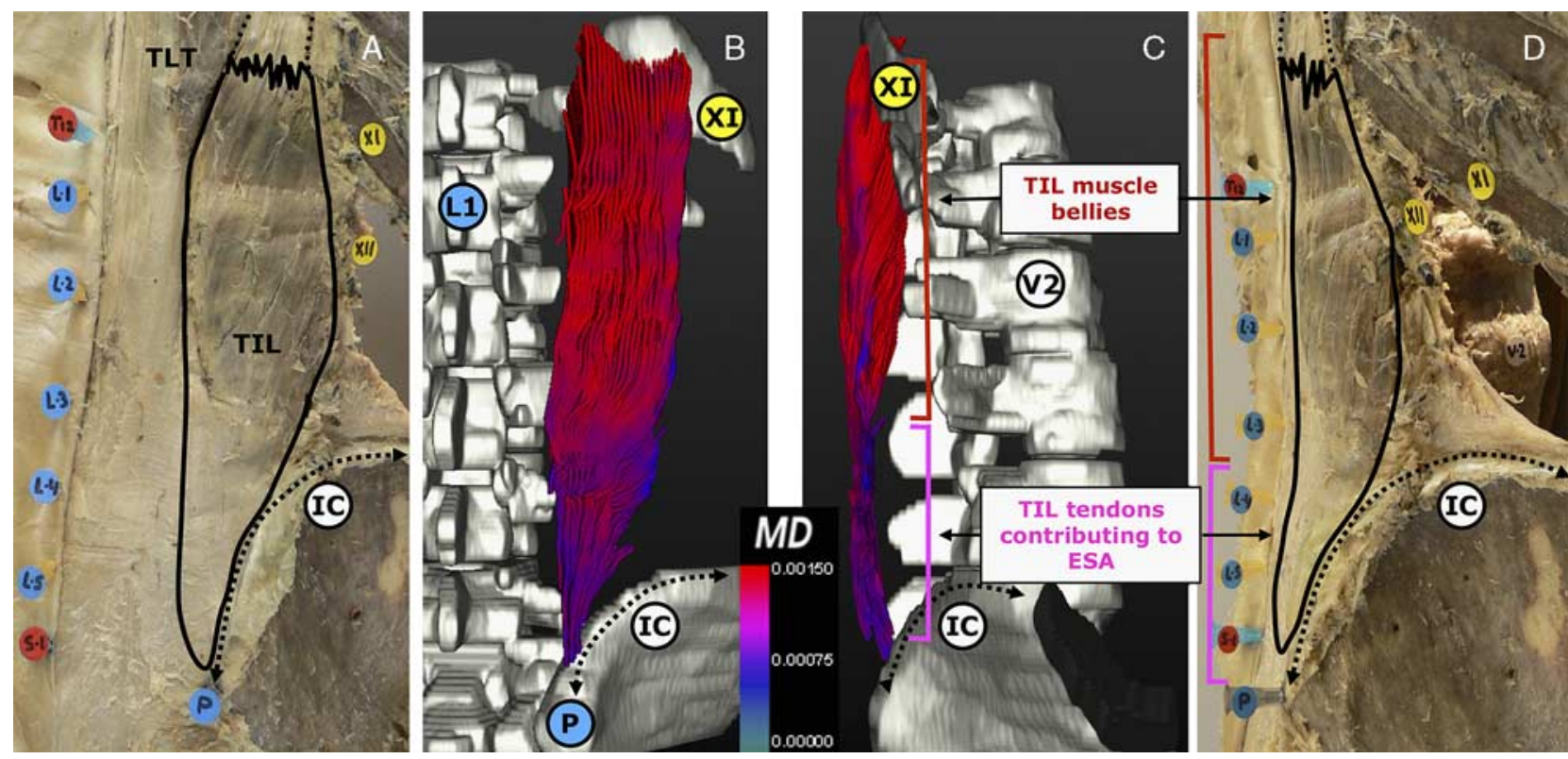

FIGURE 2. Thoracic part of iliocostalis lumborum (TIL): corresponding views of fiber tractography results and dissected cadaver specimen. A and B: Dorsal views, C and D: lateral views; all right body side. In A, B, and D spinous processes are marked T12-S1 (blue/red circles); in C and D vertebral body of second lumbar vertebra is marked V2 (white circle). Ribs XI and XII are marked with yellow circles. Iliac crest (IC) is marked with black dotted arrow; posterior superior iliac spine is marked $\mathrm{P}$ (blue circle). B and C: Diffusion tensor magnetic resonance imaging fiber tractography results of TIL are shown as colored lines (red/purple) with a background of bones; trajectories are color coded according to local diffusion coefficient (MD, $\left.\mathrm{mm}^{2} / \mathrm{s}\right)$. A and D, Solid black lines indicate contour of reconstructed part of TIL; zigzag and dotted line represent cranial continuation of TIL. Difference in level of iliac crest [L3-L4 in A and D; L5 in B and C] is due to postural differences between older healthy female (A and D) and young healthy male ( $B$ and $C)$.

tendons that collectively constitute the medial part of ESA. (Images including TLT fiber tractography results can be obtained from the corresponding author upon



FIGURE 3. Close-up of fiber tractography results of L3 and L4 fascicles of lumbar part of iliocostalis lumborum (LIL). Ventrolateral view. Diffusion tensor magnetic resonance imaging fiber tractography results are shown as colored lines (red/purple) with a background of bones; trajectories are color coded according to local diffusion coefficient (MD, $\mathrm{mm}^{2} / \mathrm{s}$ ). Trajectories of each fascicle are based on 1 region of interest drawn in the transverse plane. Transverse processes of third and fourth lumbar vertebrae are marked L3-L4 (blue circles). request.) In the dissected cadaver, TLT could not be clearly separated from LLT; the 2 ES parts were found to share cranial and caudal attachments and showed entangled muscle bellies, making it impossible to demarcate them.

\section{LLT Reconstruction}

LLT reconstructions showed few deviating trajectories (artifacts). (Images including LLT fiber tractography results can be obtained from the corresponding author upon request.)

\section{Overview of ES as a Whole}

Finally, an overview of all ES parts together was created, showing their topographical positioning and mutual relationships (Fig. 4). (Additional sets of images, which provide compilations of reconstructions viewed from several angles, are available from the corresponding author.)

\section{DISCUSSION}

The aim of the present study was to segment and reconstruct the human lumbar ES from in vivo DT-MRI measurements and to compare the results with cadaver dissection. In general, all ES parts were successfully reconstructed, although reconstructions also showed a few artifacts. DT-MRI trajectories were largely in agreement with the anatomical descriptions in literature and were confirmed by the dissection findings. However, some 

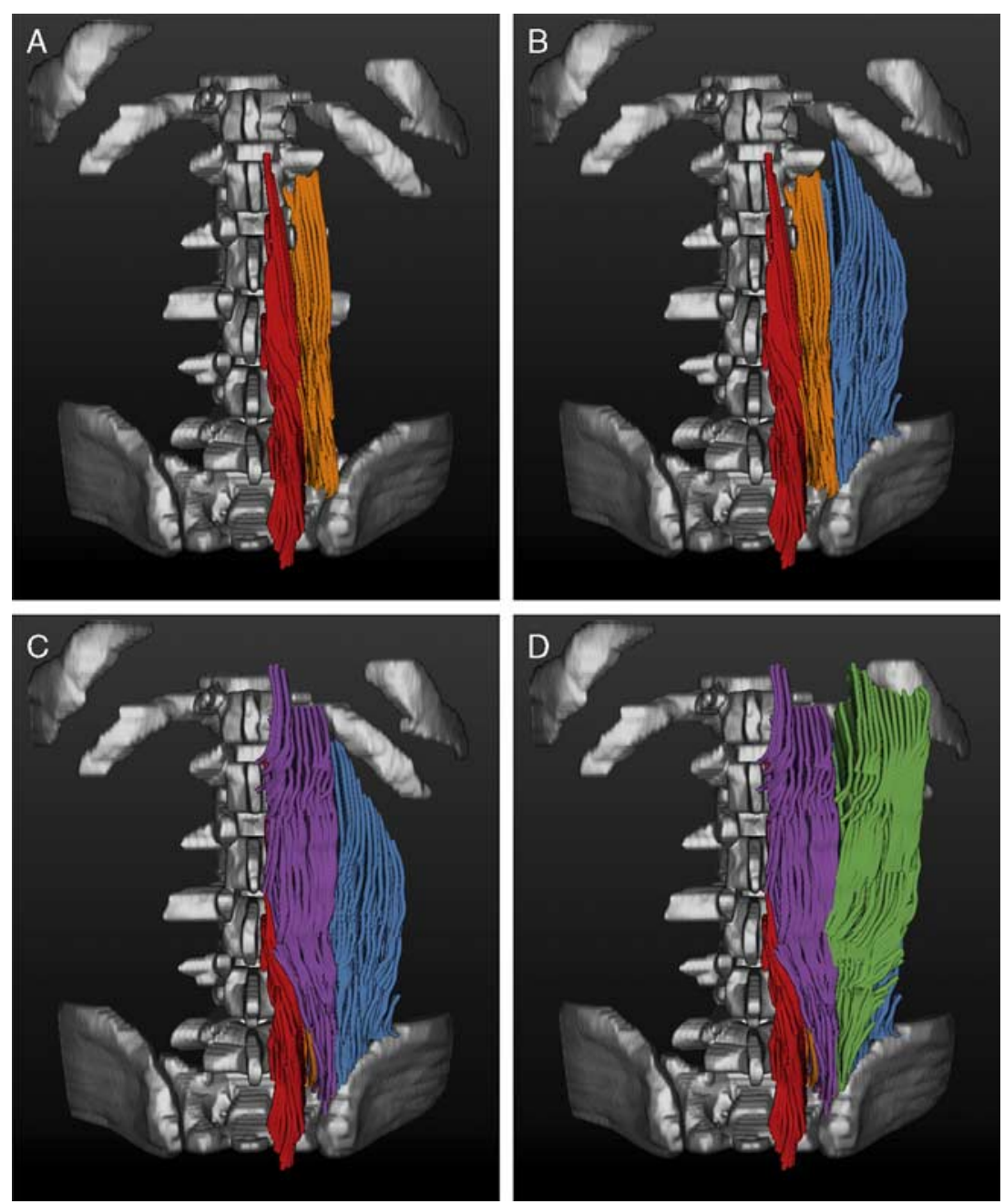

FIGURE 4. Overview of lumbar back muscle architecture (multifidus and erector spinae), dorsal view. Compilation of diffusion tensor magnetic resonance imaging muscle trajectory reconstructions showing multifidus and erector spinae parts, appended from deep to superficial, respectively, in (A) to (D). Red: multifidus, orange: lumbar part of longissimus thoracis; blue: lumbar part of iliocostalis lumborum; purple: thoracic part of longissimus thoracis; green: thoracic part of iliocostalis lumborum.

discrepancies compared with the accepted anatomical descriptions were also found.

\section{Study Limitations and Methodological Considerations}

A limitation of this study was the resolution of the measurements. DT-MRI voxels were $2 \times 2 \times 5 \mathrm{~mm}^{3}$. A problem with relatively large voxels is that several signals arising from different types of tissues and/or various muscles are represented by a single voxel, which causes the so-called partial volume effects. For reliable reconstruction of the smallest ES fascicles and details of attachments, a smaller voxel size, or an approach using higher-order models, like "high angular resolution diffusion imaging," 35 which allows the differentiation of multiple fiber orientations within a single voxel, is needed.

Another weakness is that the scan range did not include the full length of ES. Therefore, iliocostalis lumborum could not be reconstructed over its entire length.
Stitching of 2 or more image stacks, which poses a technical challenge, is needed to cover the entire span of ES. Especially stitching with more cranial thoracic stacks is difficult, because movement (due to breathing) and air/ muscle transitions (at the level of the lungs) will increase the amount of artifacts.

Furthermore, reconstructions were found to correspond with cadaver dissections to a large extent. However, differences between DT-MRI reconstructions and dissection photographs are inevitable. The volunteer for the DT-MRI measurements was a young, healthy male, whereas the dissected cadaver was that of an older, but also healthy, woman. Images may differ due to differences in posture, muscular condition, and conservation status of the cadaver. However, although muscle sizes and skeletal proportions do not match exactly, the similarities found in muscle shapes and sites of attachments are obvious.

Finally, the design of this study included only 1 healthy subject, which was compared with only 1 cadaver. 
Therefore, no conclusions can be drawn concerning the reproducibility of the findings. However, the purpose of this study was to show feasibility of the DT-MRI reconstruction method and to illustrate the possibilities for clinical application and use in spine research; this was not meant to be a study on reliability or anatomical variability.

\section{Reconstruction (in-)Accuracy}

Close-up fiber tractography results (Fig. 3) showed inaccuracies at the sites of the attachment of muscles to bones. These inaccuracies are probably due to one or more of the following reasons: the limited resolution of the DT-MRI measurements, partial volume effects, and/ or inaccurate (because manually performed) bone segmentation. Technical solutions are likely to improve the results. However, from an anatomical perspective it can be argued that the attachment of muscles to bone in reality is not a sharp, point-to-point dichotomous distinction, but merely a gradual transition from muscle fibers, through connective tissue, to the bone. This complex, functional continuity of tissues may be reflected in our reconstructions. Most likely, both technical and anatomical factors play a role in explaining the (in-) accuracy of the reconstruction details.

A similar explanation can be offered for the suboptimal modeling of longissimus thoracis. The TLT fiber reconstruction showed some unlikely caudal trajectory endings, and the reconstructed LLT appeared somewhat more slender than expected. In the dissected cadaver, TLT could not be clearly separated from LLT. This finding of actual TLT and LLT architecture not being that of 2 isolated muscles, may (in part) explain the reconstruction results. TLTs and LLTs entwined architecture may have complicated the drawing of separate ROIs for these ES parts. The experience that this muscle was hard to reconstruct in DT-MRI and difficult to distinguish in the cadaver (TLT and LLT), may not be a coincidence. In contrast, reconstruction of iliocostalis lumborum resulted in robust and reliable fiber tractography results. This latter muscle could be clearly separated and demarcated in dissection as well. It can be argued that the "imperfection" of some of the current reconstructions is not only due to technical shortcomings, but also reflects the actual anatomical complexity.

\section{Comparisons With Literature}

The reconstructions largely corresponded with the literature. However, some discrepancies and indistinct results with regard to anatomy were found as well. These discrepancies are related to topics that have been a subject of debate in literature for a long time.

In the current study, the dense connective tissue structure of ESA was found to constitute mainly from the superficial parts of ES (TIL and TLT), with the tendinous ESA overlying the deep muscle bellies of LIL and LLT. Only few dorsal fibers of LIL took part in ESA. Our findings match the descriptions by Bogduk ${ }^{25,26}$ and Macintosh and Bogduk, ${ }^{29}$ and contradict Daggfeldt ${ }^{28}$ on this issue.
Furthermore, TLT and LLT appeared difficult to distinguish in both reconstruction and dissection, which tentatively implies that both parts of longissimus thoracis should not be viewed as 2 isolated muscles. Historically, authors fairly agreed on the anatomical description of the superficial parts of ES, but opinions typically differed about how to further subdivide the deep ES. ${ }^{27,28}$ Our findings may reflect this discussion; apparently the distinction between the 4 parts of ES is not obvious.

\section{Theoretical and Clinical Relevance}

DT-MRI already has been proven useful in evaluating musculoskeletal injuries. ${ }^{36}$ From a functional perspective, a proper understanding of the ES architecture details is needed, for instance to enable biomechanical modeling of spine loading during task performance. This study shows that DT-MRI enables adequate reconstruction of the complex muscle geometry in the lumbar spine. Advances in DT-MRI technology may contribute to the further clarification of ES architecture.

As a next step, feasibility to use these reconstructions as input for estimation of muscle architecture parameters in individualized biomechanical modeling has to be proven. Such models are promising as a tool in musculoskeletal injury evaluation and for improvement of understanding of pathologies like scoliosis. A 3D biomechanical analysis of the lumbar spine could support medical doctors in diagnosis and preoperative planning, and could also provide useful information for rehabilitation. A detailed reconstruction of the in vivo lumbar muscles can also be of clinical importance to improve the understanding of low back pain mechanisms. It can be assumed that within the large group of nonspecific low back pain patients some suffer from specific muscular injuries, which cannot be revealed by current imaging techniques.

\section{CONCLUSIONS}

Anatomically accurate reconstruction of ES architecture using DT-MRI is possible. With this rapidly evolving technology, in the near future DT-MRI muscle reconstructions may become useful in individualized spinal modeling for clinical and/or research purposes.

\section{ACKNOWLEDGMENTS}

The authors thank Johan Hekking and Leon Huibers for their technical assistance with dissection and photography.

\section{APPENDIX}

Technical details of MRI data acquisition

(1) T1-weighted turbo spin echo (TSE): reconstructed voxel dimensions $0.5 \times 0.5 \times 5.0 \mathrm{~mm}^{3}$; repetition time (TR)/echo time (TE) $550 / 12 \mathrm{~ms}$; number of signal averages 2 .

(2) DT-MRI: A diffusion-weighted spin echo single-shot planar imaging sequence (DW-SE-EPI) was used. Reconstructed voxel dimensions $2 \times 2 \times 5 \mathrm{~mm}^{3}$; field of view $224 \times 224 \mathrm{~mm}^{2}$; acquisition matrix $112 \times 112$; number of signal averages 2 ; number of diffusion gradient directions 15; TR/TE $1100 / 41 \mathrm{~ms}$; B-value $400 \mathrm{~s} / \mathrm{mm}^{2}$; fat suppression SPAIR. 
(3) Dual echo gradient echo sequence (GE): Reconstructed voxel dimensions $2 \times 2 \times 5 \mathrm{~mm}^{3}$; number of signal averages $2 ; \mathrm{TR} / \mathrm{TE}_{1} / \mathrm{TE}_{2}$ 12/4.6/9.6 ms.

\section{REFERENCES}

1. Lansdown DA, Ding Z, Wadington M, et al. Quantitative diffusion tensor MRI-based fiber tracking of human skeletal muscle. $J$ Appl Physiol. 2007;103:673-681.

2. Sinha S, Sinha U, Edgerton VR. In vivo diffusion tensor imaging of the human calf muscle. $J$ Magn Reson Imaging. 2006;24: 182-190.

3. Heemskerk AM, Sinha TK, Wilson KJ, et al. Quantitative assessment of DTI-based muscle fiber tracking and optimal tracking parameters. Magn Reson Med. 2009;61:467-472.

4. Froeling M, Nederveen AJ, Heijtel DF, et al. Diffusion-tensor MRI reveals the complex muscle architecture of the human forearm. J Magn Reson Imaging. 2012;36:237-248.

5. Lieber RL. Chapter 1: skeletal muscle anatomy. In: Lieber RL, ed. Skeletal Muscle Structure, Function, and Plasticity: the Physiological Basis of Rehabilitation. 3rd ed. Philadelphia: Wolters Kluwer/ Lippincott Williams \& Wilkins; 2010:1-40.

6. Battermann N, Appell HJ, Dargel J, et al. An anatomical study of the proximal hamstring muscle complex to elucidate muscle strains in this region. Int J Sports Med. 2011;32:211-215.

7. Blemker SS, Delp SL. Three-dimensional representation of complex muscle architectures and geometries. Ann Biomed Eng. 2005;33: 661-673.

8. Blemker SS, Pinsky PM, Delp SL. A 3D model of muscle reveals the causes of nonuniform strains in the biceps brachii. $J$ Biomech. 2005;38:657-665.

9. Lieber RL, Friden J. Functional and clinical significance of skeletal muscle architecture. Muscle Nerve. 2000;23:1647-1666.

10. Opar DA, Williams MD, Shield AJ. Hamstring strain injuries: factors that lead to injury and re-injury. Sports Med. 2012;42: 209-226.

11. Lieber RL, Friden J. Implications of muscle design on surgical reconstruction of upper extremities. Clin Orthop Relat Res. 2004; 267-279.

12. Strijkers G, Drost M, Nicolay K. Chapter 41: diffusion imaging in muscle. In: Jones J, ed. Diffusion MRI: Theory, Methods and Applications. New York: Oxford University Press; 2011:672-689.

13. Mori S, van Zijl PC. Fiber tracking: principles and strategies - a technical review. NMR Biomed. 2002;15:468-480.

14. Junqueira L, Carneiro J. Muscle tissue. In: Junqueira L, Carneiro J, eds. Basic Histology Text and Atlas. 10th ed. New York: Lange Medical Books; 2003:191-213.

15. Galban CJ, Maderwald S, Uffmann K, et al. Diffusive sensitivity to muscle architecture: a magnetic resonance diffusion tensor imaging study of the human calf. Eur J Appl Physiol. 2004;93: 253-262.

16. Galban CJ, Maderwald S, Uffmann K, et al. A diffusion tensor imaging analysis of gender differences in water diffusivity within human skeletal muscle. NMR in Biomedicine. 2005;18: 489-498.
17. Karampinos DC, King KF, Sutton BP, et al. Myofiber ellipticity as an explanation for transverse asymmetry of skeletal muscle diffusion MRI in vivo signal. Ann Biomed Eng. 2009;37:2532-2546.

18. Van Donkelaar CC, Kretzers LJ, Bovendeerd PH, et al. Diffusion tensor imaging in biomechanical studies of skeletal muscle function. J Anat. 1999;194(pt 1):79-88.

19. Heemskerk AM, Strijkers GJ, Vilanova A, et al. Determination of mouse skeletal muscle architecture using three-dimensional diffusion tensor imaging. Magn Reson Med. 2005;53:1333-1340.

20. Budzik JF, Le Thuc V, Demondion X, et al. In vivo MR tractography of thigh muscles using diffusion imaging: initial results. Eur Radiol. 2007;17:3079-3085.

21. Froeling M, Oudeman J, van den Berg S, et al. Reproducibility of diffusion tensor imaging in human forearm muscles at $3.0 \mathrm{~T}$ in a clinical setting. Magn Reson Med. 2010;64:1182-1190.

22. Levin DI, Gilles B, Madler B, et al. Extracting skeletal muscle fiber fields from noisy diffusion tensor data. Med Image Anal. 2011;15:340-353.

23. Zijta FM, Froeling M, van der Paardt MP, et al. Feasibility of diffusion tensor imaging (DTI) with fibre tractography of the normal female pelvic floor. Eur Radiol. 2011;21:1243-1249.

24. Goh V, Tam E, Taylor NJ, et al. Diffusion tensor imaging of the anal canal at 3 tesla: feasibility and reproducibility of anisotropy measures. J Magn Reson Imaging. 2012;35:820-826.

25. Bogduk N. A reappraisal of the anatomy of the human lumbar erector spinae. J Anat. 1980;131:525-540.

26. Bogduk N. The lumbar muscles and their fasciae. In: Bogduk N, ed. Clinical Anatomy of the Lumbar Spine and Sacrum. 4th ed. Edinburgh: Elsevier Churchill Livingstone; 2005:97-121.

27. Bustami FM. A new description of the lumbar erector spinae muscle in man. J Anat. 1986;144:81-91.

28. Daggfeldt K, Huang QM, Thorstensson A. The visible human anatomy of the lumbar erector spinae. Spine. 2000;25:2719-2725.

29. Macintosh JE, Bogduk N. 1987 Volvo award in basic science. The morphology of the lumbar erector spinae. Spine. 1987;12:658-668.

30. Wolfram Research Inc.. Wolfram Mathematica 7 [Computer Program]. Champaign, IL: Wolfram Research Inc.; 2008.

31. Vilanova A, Berenshot G, van de Pul C. DTI visualisation with streamsurfaces and evenly-spaced volume seeding. Proceedings of the VisSym Joint Eurographics/IEEE TCVG Symposium on Visualization Konstanz, Konstanz, Germany, May 19-21, 2004.

32. Biomedical Image Analysis Group, Eindhoven University of Technology (project leader: Anna Vilanova). DTITool - vIST/e [Computer Program]. Eindhoven, The Netherlands. http://bmia. bmt.tue.nl/software/viste/.

33. Bol GH, Kotte AN, van der Heide UA, et al. Simultaneous multimodality ROI delineation in clinical practice. Comput Methods Programs Biomed. 2009;96:133-140.

34. Bol GH. Volume Tool [Computer Program]. Utrecht, The Netherlands: University Medical Center Utrecht; 2009.

35. Tuch DS, Reese TG, Wiegell MR, et al. High angular resolution diffusion imaging reveals intravoxel white matter fiber heterogeneity. Magn Reson Med. 2002;48:577-582.

36. Zaraiskaya T, Kumbhare D, Noseworthy MD. Diffusion tensor imaging in evaluation of human skeletal muscle injury. J Magn Reson Imaging. 2006;24:402-408. 